\title{
Pregnancy and lactation interfere with the response of autoimmunity to modulation of gut microbiota
}

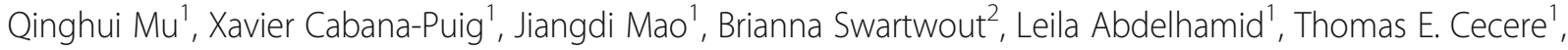 \\ Haifeng Wang ${ }^{3}$, Christopher M. Reilly ${ }^{1,4}$ and Xin M. Luo ${ }^{1 *}$ (D)
}

\begin{abstract}
Background: Dysbiosis of gut microbiota exists in the pathogenesis of many autoimmune diseases, including systemic lupus erythematosus (lupus). Lupus patients who experienced pregnancy usually had more severe disease flares post-delivery. However, the possible role of gut microbiota in the link between pregnancy and exacerbation of lupus remains to be explored.

Results: In the classical lupus mouse model MRL/Ipr, we compared the structures of gut microbiota in pregnant and lactating individuals vs. age-matched naïve mice. Consistent with studies on non-lupus mice, both pregnancy and lactation significantly changed the composition and diversity of gut microbiota. Strikingly, modulation of gut microbiota using the same strategy resulted in different disease outcomes in postpartum (abbreviated as "PP," meaning that the mice had undergone pregnancy and lactation) vs. control (naïve; i.e., without pregnancy or lactation) MRL/lpr females; while vancomycin treatment attenuated lupus in naïve mice, it did not do so, or even exacerbated lupus, in PP mice. Lactobacillus animalis flourished in the gut upon vancomycin treatment, and direct administration of $L$. animalis via oral gavage recapitulated the differential effects of vancomycin in PP vs. control mice. An enzyme called indoleamine 2,3dioxygenase was significantly inhibited by L. animalis; however, this inhibition was only apparent in PP mice, which explained, at least partially, the lack of beneficial response to vancomycin in these mice. The differential production of immunosuppressive IL-10 and proinflammatory IFNy in PP vs. control mice further explained why the disease phenotypes varied between the two types of mice bearing the same gut microbiota remodeling strategy.
\end{abstract}

Conclusions: These results suggest that pregnancy and lactation interfere with the response of autoimmunity to modulation of gut microbiota. Further studies are necessary to better understand the complex relationship between pregnancy and lupus.

Keywords: Lupus, Pregnancy, Gut microbiota, Lactobacillus animalis, IDO, Treg, IFNy, Leaky gut

\section{Background}

Autoimmune diseases occur when individuals have disturbances in immune responses, especially $\mathrm{T}$ and $\mathrm{B}$ cell responses, which in turn attack self-antigens and subsequently cause tissue damage [1]. Comprised of more than 70 different disorders, autoimmune diseases affect more than 7\% of the general population [2]. Many of these diseases are more prevalent in females [3]. Sex hormones

\footnotetext{
* Correspondence: xinluo@vt.edu

'Department of Biomedical Sciences and Pathobiology, College of Veterinary Medicine, Virginia Tech, Blacksburg, VA, USA

Full list of author information is available at the end of the article
}

contribute to this phenomenon by regulating the immune system via hormone receptors [4]. During pregnancy, to support fetal development, the maternal body undergoes changes on hormones, immunity, and metabolism [5]. Evidences suggest that there is an association between pregnancy and autoimmune diseases [3, 6]. However, except for hormonal effects, it remains unclear how pregnancy influences the autoimmunity [7].

Systemic lupus erythematosus, or lupus, is a prototypical systemic autoimmune disease characterized by severe and persistent multiorgan inflammation [8]. The loss of self-tolerance and activation of autoreactive immune cells

(c) The Author(s). 2019 Open Access This article is distributed under the terms of the Creative Commons Attribution 4.0 International License (http://creativecommons.org/licenses/by/4.0/), which permits unrestricted use, distribution, and 
lead to the production of autoantibodies and diverse proinflammatory cytokines, pathogenic cell infiltration, and subsequent tissue damage in multiple organs, including, but not limited to, the skin, kidney, lung, heart, and brain [9]. With more than half of patients affected, kidney inflammation, or lupus nephritis, is the leading cause of lupus mortality [10]. Although affecting both females and males, lupus is a highly gender-biased disease with about 9 times more incidences in females of child-bearing age. Pregnant women with lupus have a higher risk of lupus flares post-delivery [11]. Therefore, some clinicians recommend women with lupus to avoid pregnancy [12-15]. A better understanding of the mechanisms underlying pregnancy-induced disease flares and the development of new therapeutic strategies for pregnant women will likely benefit these patients for successful pregnancies and better lupus outcomes.

The exact mechanism of pathogenesis is still unclear for lupus, but genetics, hormones, and environmental factors have been suggested as the major causes [8]. As an important factor linking environmental factors with the host, gut microbiota and its interaction with host health and disease have drawn a lot of attention in the past decade [16]. Increasing evidences indicate that a perturbed gut microbiota is associated with multiple autoimmune disorders such as lupus [17]. On the one hand, dysbiosis of gut microbiota does exist in both human patients and lupus-prone mouse models [18-20]. On the other hand, remodeling of gut microbiota community by antibiotics and probiotics has been shown to efficiently modulate disease development in lupus-prone mice [21-23]. These evidences have demonstrated the involvement of gut microbiota in lupus pathogenesis and suggested modulating gut microbes as a potential therapeutic method. Three potential mechanisms may explain the role of gut microbiota in lupus: (1) a leaky gut and associated bacterial translocation [23-25], (2) molecular mimicry [26], and (3) induction of regulatory immune response [22]. But additional mechanisms may exist. For example, the capability of gut microbiota to interact with host hormones and molecules produced by microbes, such as short chain fatty acids (SCFAs), can potentially influence lupus pathogenesis $[27,28]$. Furthermore, despite a higher risk of lupus flares in pregnant patients post-delivery $[11,15]$, the role of gut microbiota in the link between pregnancy and exacerbated lupus has never been examined.

The current study is driven by an interesting findingthat vancomycin treatment attenuated lupus nephritis in naïve MRL/lpr females [21] but somehow exacerbated the disease in MRL/lpr mice that had experienced pregnancy and lactation (named postpartum or "PP" mice). In contrast to downregulating IL-6 and IL-17 production in control or naïve mice, the oral antibiotic was not able to affect either IL-6 or IL-17 expression in PP mice. Instead, in PP mice, oral vancomycin administration resulted in reduced regulatory $\mathrm{T}$ (Treg) and IL-10 producing B cell (B10) responses and subsequently a dampened antiinflammatory IL-10 response. At the same time, the production of proinflammatory IFN $\gamma$ was elevated. Importantly, the imbalance of IL-10 and IFN $\gamma$ occurred only in PP but not naïve MRL/lpr mice. This provides an explanation of the differed disease manifestations between these two types of mice receiving the same vancomycin treatment. As pregnancy and lactation can significantly change the gut microbiota diversity and composition [29], and that vancomycin is not absorbed in gastrointestinal tract and thus not systemic [30], we analyzed the local gut microbiota status. As anticipated, vancomycin removed the majority of bacteria and greatly increased the relative abundance of Lactobacillus spp. [31, 32], particularly the species Lactobacillus animalis. We next determined whether weekly $L$. animalis oral gavage would recapitulate the differential effects of vancomycin on lupus disease in control vs. PP MRL/lpr mice. Indeed, direct administration of $L$. animalis significantly worsened lupus disease in PP mice. Consistently, L. animalis also decreased IL-10 but increased IFNy. Further investigations showed that an enzyme indoleamine 2,3-dioxygenase (IDO) was inhibited by the enriched $L$. animalis; however, this inhibition was only apparent in PP mice. As IDO is known to be able to activate Treg cells [33-35], this observation may be able to explain the differential effects of vancomycin in control vs. PP mice. Together, these results provide a potential mechanism by which pregnancy and lactation may interfere with the response of autoimmunity to modulation of gut microbiota.

\section{Results}

\section{Lack of beneficial response to oral vancomycin in PP MRL/Ipr mice}

In our previous study, lupus disease was greatly attenuated in female MRL/lpr mice receiving oral vancomycin from 9 weeks to 15 weeks of age (the endpoint) [21]. However, when the exactly same treatment procedure was implemented on PP MRL/lpr females (Fig. 1a), the beneficial effect was not observed (Fig. 1b-e). As reported previously, vancomycin administration significantly decreased splenomegaly and the size of mesenteric lymph nodes (MLN) in age-matched naïve MRL/lpr mice (labeled "CTL"). On the contrary, the antibiotic treatment worsened splenomegaly in PP mice (Fig. 1b). The level of anti-DNA antibodies, a hallmark of disease in both lupus patients and lupusprone animal models $[8,36]$, was significantly reduced by vancomycin in naïve mice but not PP mice (Fig. 1c and Additional file 1: Figure S1A). Notably, the level of this autoantibody was even increased in vancomycin-treated PP mice when they were analyzed at 11 weeks of age and 


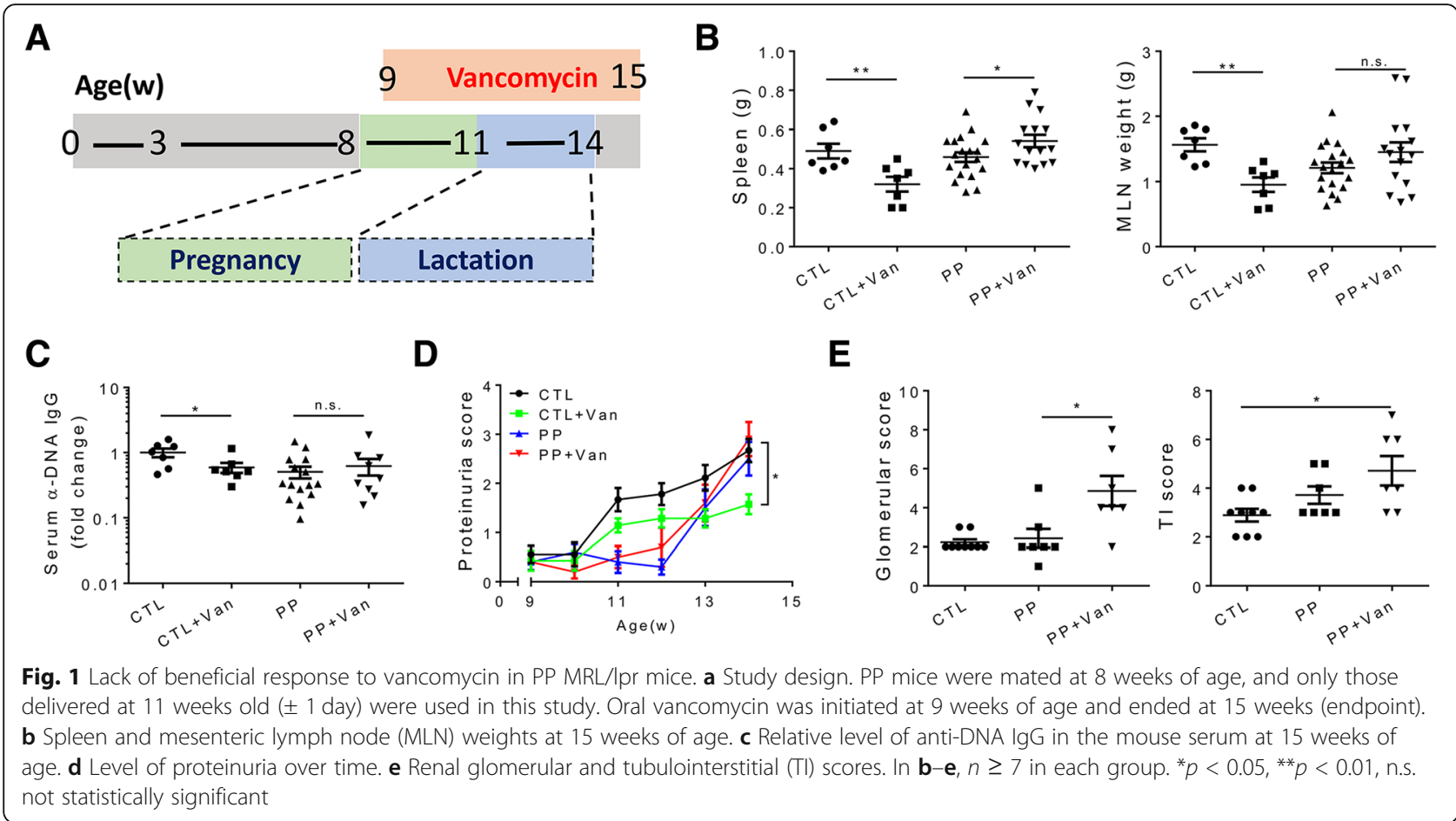

at the time of delivery (Additional file 1: Figure S1B). To assess kidney inflammation and function, we measured the level of proteinuria weekly and had the kidney sliced and scored by a pathologist. In control mice, the proteinuria level was significantly decreased by vancomycin treatment (Fig. 1d). Correspondingly, the glomerular and tubulointerstitial (TI) scores were both lowered (data not shown and [21]). However, in PP mice, the proteinuria level was not influenced by vancomycin (Fig. 1d). Interestingly, the level of proteinuria decreased around the time pups were delivered and quickly caught up afterwards both with and without vancomycin treatment. Renal injury was more severe in vancomycin-treated PP mice as suggested by significantly higher kidney histopathological scores (Fig. 1e). Taken together, these data indicate that contrary to the protective effect on naïve mice, vancomycin treatment was not beneficial but detrimental in MRL/lpr mice that had experienced pregnancy and lactation.

\section{Vancomycin-mediated downregulation of regulatory immune response in PP MRL/lpr mice}

We next sought to explore the underlying mechanism(s) behind the phenomenon that the same antibiotic treatment led to opposite disease outcomes in control vs. PP mice. We examined different immune cell populations and diverse inflammatory mediators, especially those related to IL-17 (Additional file 1: Figure S2A), as the production of this cytokine was ameliorated in naïve mice receiving vancomycin [21]. IL-6 is known as an important pathogenic cytokine in both human and mouse lupus due to, at least partially, its ability to induce Th17 cell differentiation and IL-17 production [37, 38]. In age-matched control mice, oral vancomycin significantly reduced IL-6 level and subsequently resulted in lower IL-17 production from different cellular resources (Fig. 2a-d and [21]). In contrast, the circulating IL- 6 in PP mice remained unchanged after vancomycin treatment (Fig. 2a). In addition, although $\mathrm{CD}^{+}$and $\mathrm{CD} 8^{+} \mathrm{T}$ cells were not affected (Fig. $2 \mathrm{~b}$ and Addditional file 1: Figure S2B), the percentage of double negative $\mathrm{T}$ (DN-T) cells in the spleen was changed by vancomycin in both control and PP mice, but in an opposite fashion (Fig. 2b). In both lupus patients and mouse models, DN-T cells expand and emerge as a major IL-17 producer [39, 40]. Remarkably, in control mice, vancomycin treatment not only reduced the percentage of DN$\mathrm{T}$ cells but also reduced the proportion of $\mathrm{DN}-\mathrm{T}$ cell capable of secreting IL-17 (Additional file 1: Figure S2C). It was a different story for PP MRL/lpr mice. Pregnancy and lactation significantly increased the percentage of DN-T cells (Fig. 2b), but it appears that the experiences dampened the capability of DN-T cells to secrete IL-17, which was not further changed by vancomycin (Additional file 1 : Figure S2C). Apart from DN-T cells, we also investigated Th17 cells which secrete IL-17 as the signature cytokine [41]. Interestingly, oral vancomycin leads to a similar decrease of Th17 cells in both control and PP mice (Fig. 2d). Considering the differed DN-T and Th17 responses in PP mice upon vancomycin treatment, we analyzed the total IL-17 expressing $\mathrm{T}$ cells (Fig. 2c). While vancomycin 


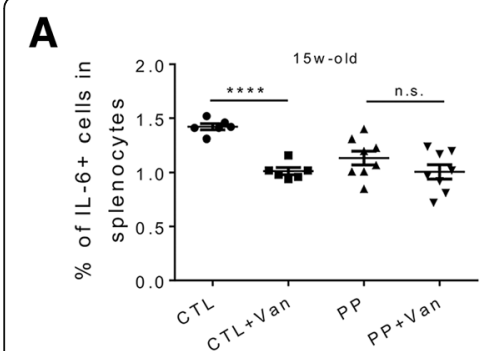

C

Gated on CD3+ cells
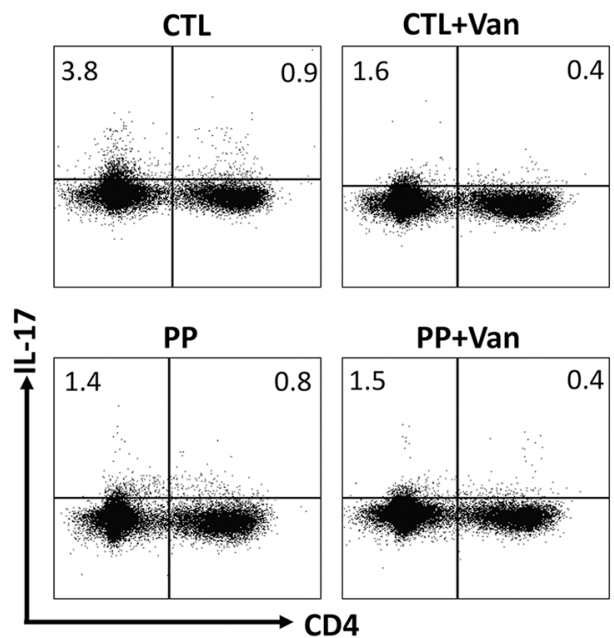

F

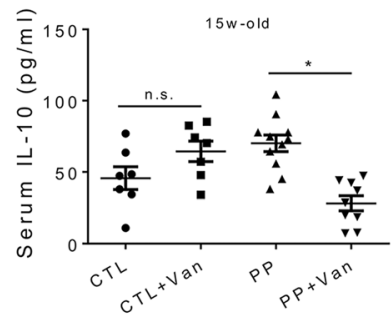

B

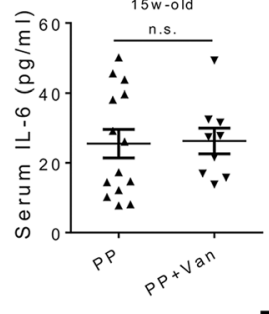

D
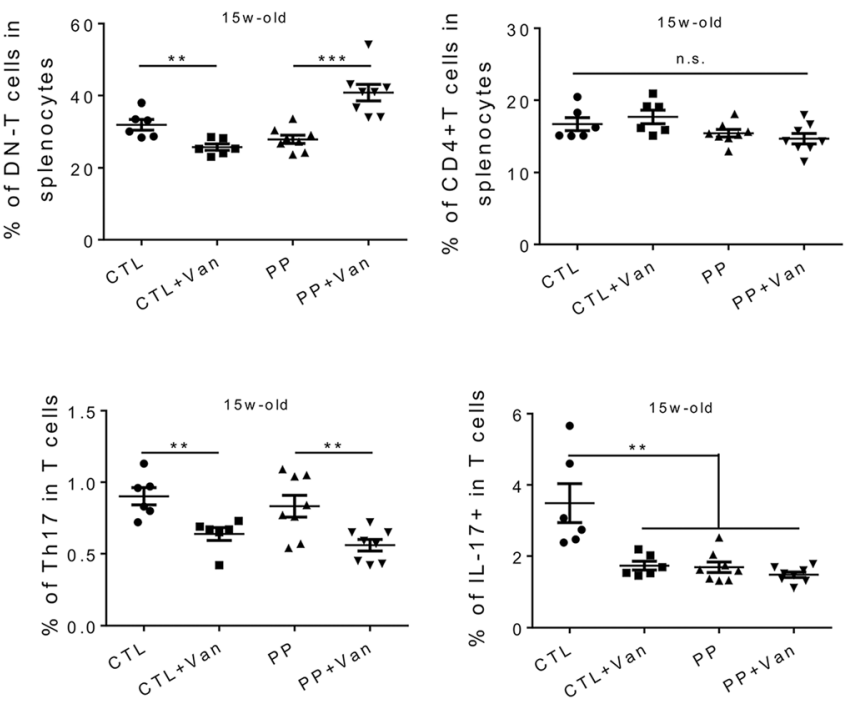

E
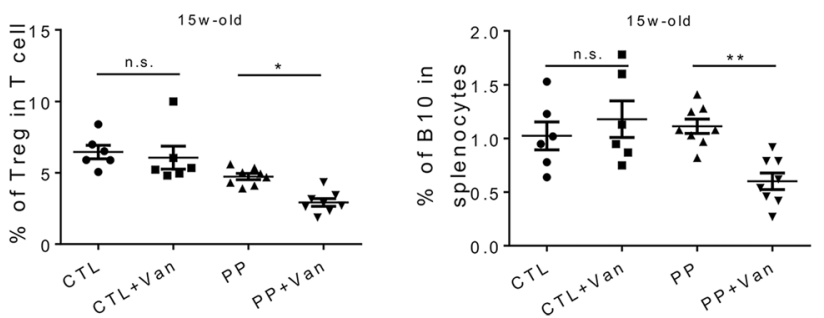

G

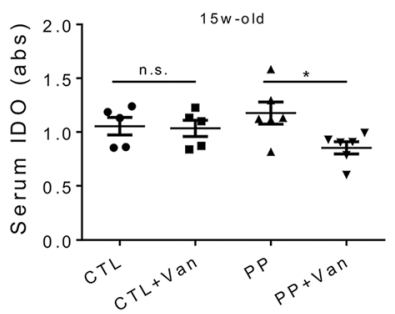

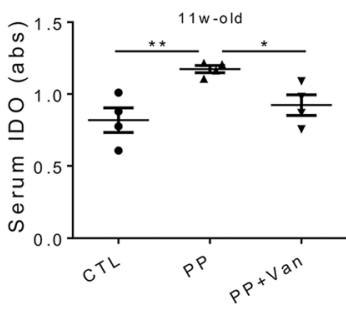

Fig. 2 Vancomycin-mediated downregulation of regulatory immune response in PP MRL/Ipr mice. a Left: percentage of IL-6 producing cells in

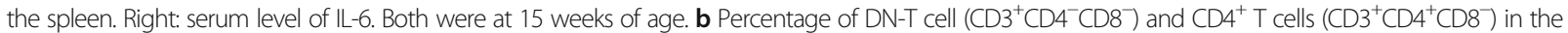
spleen at the 15 weeks of age ( $n \geq 6$ per group). c Representative FACS plots of IL-17 producing T cells. $\mathbf{d}$ Percentage of Th17 cells and IL-17 producing T cells $\left(C D 3^{+} I L-17^{+}\right)$in the spleen at the 15 weeks of age ( $n \geq 6$ per group). e Percentage of Treg $\left(\mathrm{CD}^{+} \mathrm{CD}^{+} \mathrm{Foxp}^{+}\right)$and B10 $\left(\mathrm{CD} 19^{+} \mathrm{IL}-10^{+}\right)$cells in the spleen at the 15 weeks of age ( $n \geq 6$ per group). $\mathbf{f}$ Level of $\mathrm{LL}-10$ in mouse serum at the 15 weeks of age ( $n \geq 7$ per group). $\mathbf{g}$ Level of IDO in mouse serum at the 15 weeks and 11 weeks of age ( $n \geq 4$ per group). ${ }^{*} p<0.05,{ }^{* *} p<0.01,{ }^{* * *} p<0.001,{ }^{* * *} p<0.0001$, n.s. not statistically significant

significantly downregulated the percentage of IL-17 expressing $\mathrm{T}$ cells in control mice, PP mice exhibited similar IL-17 producing abilities among all $\mathrm{T}$ cells with or without vancomycin (Fig. 2d). It is noteworthy that these changes were evident in the spleen, but not in the mesenteric lymph node (MLN; data not shown). Taken together, these results indicate that vancomycin treatment led to dampened IL-6 and IL17 production and attenuated lupus nephritis in control mice but failed to change either the IL-17 response or disease manifestations in PP mice.
Opposing the pro-inflammatory function of IL-17 in autoimmune lupus, Treg cells play a central regulatory role in suppressing disease development and progression $[42,43]$. We thus measured splenic $\mathrm{CD}^{+}{ }^{+} \mathrm{Foxp}^{+}{ }^{+}$Treg cells (Additional file 1: Figure S2A) and found that the vancomycin treatment resulted in a significant decrease of Treg cells in PP but not control mice (Fig. 2e). We also assessed the percentage of CD19+ $\mathrm{IL}-10^{+}$cells in the spleen, which are B10 cells that have emerged as an important cell type regulating diverse autoimmune disorders [44]. A similar decrease of B10 cells was noted in PP but not 
control mice (Fig. 2e). As a result, the serum IL-10 concentration was significantly reduced in PP mice receiving vancomycin, but remained unchanged in control mice (Fig. 2f). As both regulatory B (Breg) cells and Treg cells can secrete IL-35, another important anti-inflammatory cytokine [45], we measured the serum level of IL-35. Surprisingly, IL-35 was not changed with or without vancomycin treatment (Additional file 1: Figure S2D), which was not consistent with the observed downregulation of Treg and B10 cells. It suggests that other IL-35 producing cells may exist in MRL/lpr mice [46].

Next, we set to evaluate the factor(s) contributing to the differential outcomes of regulatory cells and IL-10 production in control vs. PP mice. During pregnancy, the maternal immune system is dampened to tolerate and accept the fetus [47]. Different mechanisms are involved in pregnancy-induced immune tolerance, and IDO is one of them [48]. IDO is capable of promoting Treg differentiation and therefore plays a role in regulating autoimmune diseases [33, 34]. At 11 weeks of age (the time of delivery), PP MRL/lpr females had significantly higher IDO expression compared to age-matched naïve mice (Fig. $2 \mathrm{~g}$ ), although the increase of IDO was not apparent at 15 weeks of age when the pups had been weaned. Consistent with the observed changes for Treg cells (Fig. 2e) and serum IL-10 (Fig. 2f), vancomycin treatment significantly reduced the serum level of IDO only in PP mice (Fig. 2g). This observation provides a possible explanation for the differential effects of vancomycin on lupus disease in control vs. PP mice.

\section{Identification of a bacterium responsible for the differential effects of vancomycin on control vs. PP MRL/ Ipr mice}

It is well established that pregnancy can influence gut microbiota community [29, 49]. However, the possible role of gut microbiota in the link between pregnancy and lupus flares remains uncovered. In MRL/lpr lupusprone mice, we found that compared to age-matched control females, PP mice had modest increases of gut microbiota diversity and richness during pregnancy and lactation (Fig. 3a). The composition of gut microbiota changed as well (Fig. 3b). During pregnancy, at the phylum level, only Firmicutes was significantly increased (Fig. 3c). However, during lactation, Verrucomicrobia was greatly increased in the gut of PP mice (Additional file 1: Figure S3A). Under the phylum Firmicutes, Clostridiales and Lactobacillales significantly increased during pregnancy while Erysipelotrichales significantly decreased (Fig. 3d, e). In addition, at the order level, the relative abundance of Bacteroidales, Acholeplasmatales, Verrucomicrobiales, and Desulfovibrionales was all significantly changed during either pregnancy or lactation (Additional file 1: Figure S3C). Recently, a Lachnospiraceae species, Ruminococcus gnavus, has been implicated in lupus nephritis [50]. However, we did not observe statistically significant differences in either the Lachnospiraceae family or $R$. gnavus. While many bacterial species in the gut microbiota are able to produce SCFAs, primarily butyrate, acetate, and propionate [28], the variations of gut microbiota in control vs. PP mice did not influence the levels of SCFAs, although slightly more butyrate was noted in the feces of PP mice (Fig. 3f).

For mice receiving vancomycin, the changes of gut microbiota in control and PP mice were similar: Firmicutes, in particular Lactobacillales, flourished (Fig. 3c, e). Further analysis showed that one Lactobacillus species, L. animalis, accounted for the vast majority of Lactobacillales in vancomycin-treated mice (Fig. $3 \mathrm{e}$ and Additional file 1: Figure S3B). It is worth mentioning that the total bacterial load in the gut reduced more than $90 \%$ with vancomycin treatment (data not shown) As a result, the levels of 3 major SCFAs in the feces dramatically decreased (Fig. 3f). Conversely, the fecal heptanoic acid increased although the cause is unclear.

We decided to investigate the role of $L$. animalis as it dominated the gut microbiota of vancomycin-treated mice. While it belongs to a family with numerous species bearing probiotic properties, studies on $L$. animalis are limited and its influence on host biology is unknown. To test if the enriched $L$. animalis was responsible for the differential effects observed for vancomycin, we performed an experiment involving 4 groups: naïve mice gavaged with PBS (CTL + PBS) or L. animalis $(\mathrm{CTL}+$ L.ani), and PP mice gavaged with PBS (PP + PBS) or $L$. animalis $(\mathrm{PP}+$ L.ani). The weekly bacteria oral gavage was initiated from 9 weeks of age and lasted until 15 weeks of age, replacing vancomycin in the experimental design shown in Fig. 1a. Strikingly, the L. animalis gavage led to worsened splenomegaly in PP but not control mice (Fig. 3g). Neither the MLN weight nor the level of anti-DNA IgG in the circulation was changed (Fig. 3g, h), consistent with what was observed with vancomycin treatment (Fig. 1b, c). Furthermore, the bacteria gavage significantly worsened proteinuria only in PP mice (Fig. 3i). These results suggest that $L$. animalis recapitulated the effects of vancomycin and was able to exacerbate lupus nephritis in PP mice rather than naïve mice.

\section{Mechanisms behind the differential effects of vancomycin and $L$. animalis in control vs. PP MRL/Ipr mice Microbial translocation}

Microbial translocation, caused by a leaky gut, has been recognized as an important factor affecting multiple autoimmune disorders including lupus [24, 51, 52]. In lupus-prone MRL/lpr mice, we found that a mixture of probiotics could reduce gut leakiness leading to attenuation of lupus nephritis [22]. In human lupus patients, the circulating endotoxin was highly elevated indicating a 


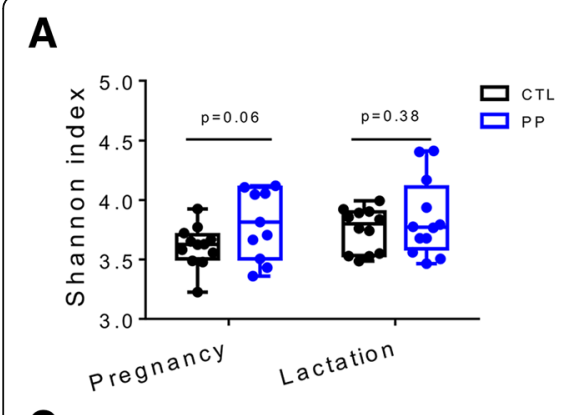

C

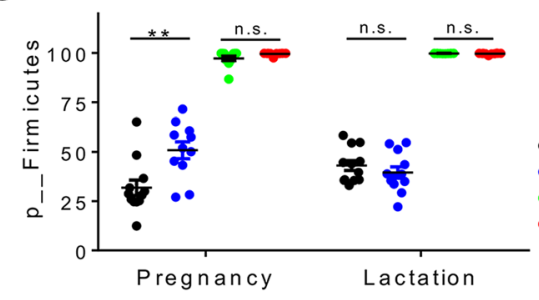

E

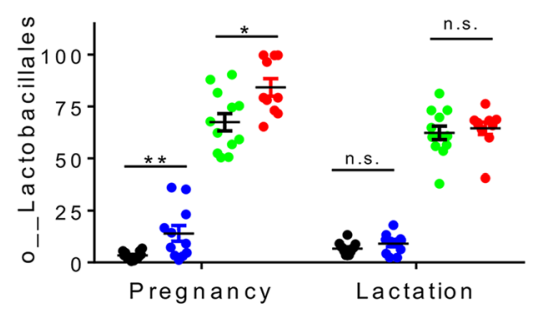

G

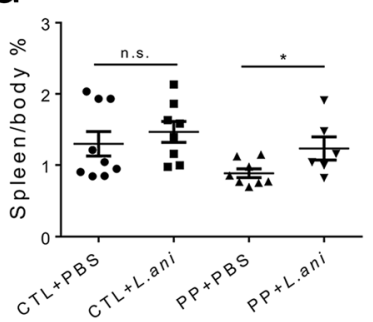

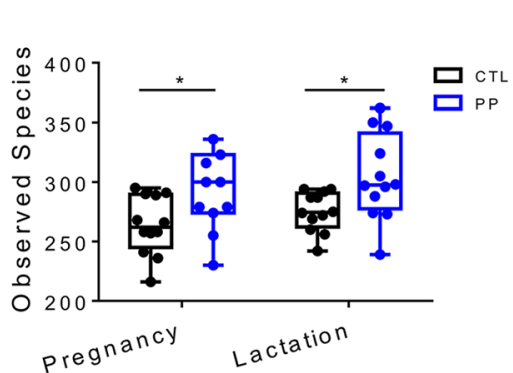

D
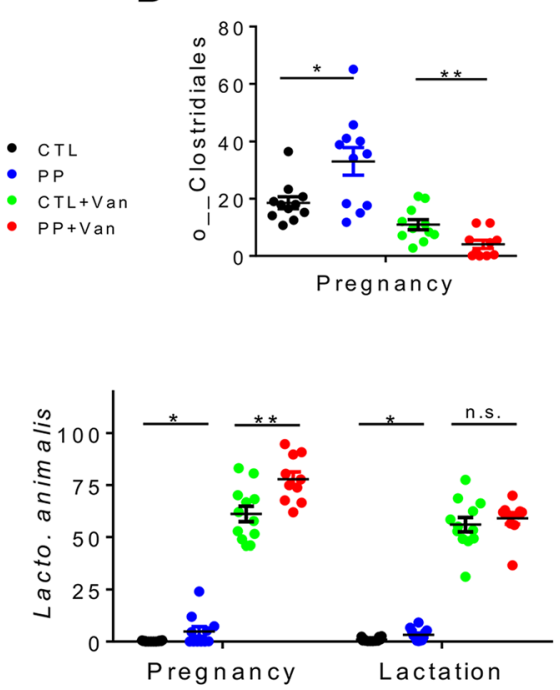

H

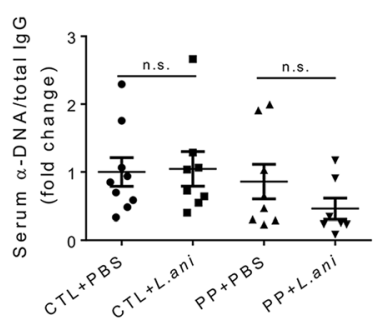

B
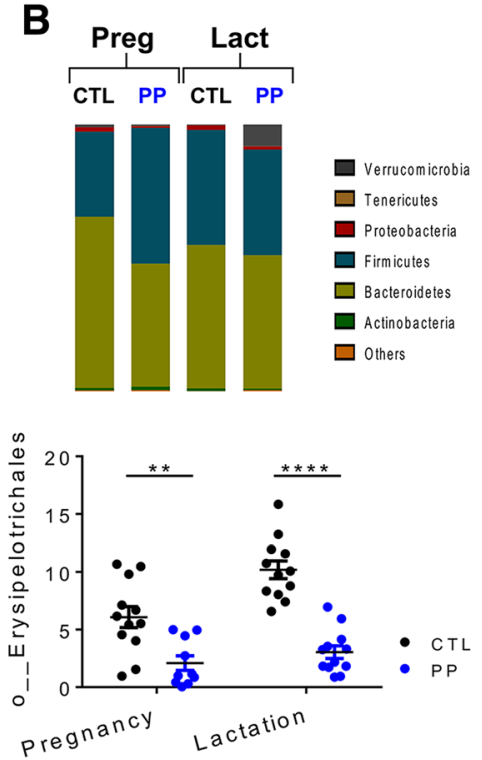

$\mathbf{F}$

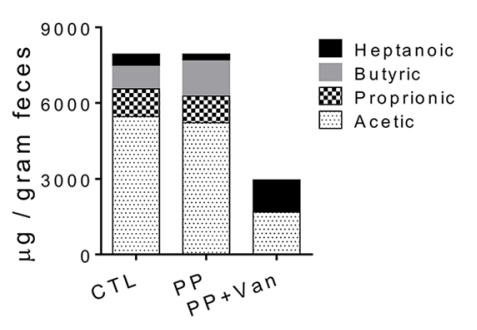

I

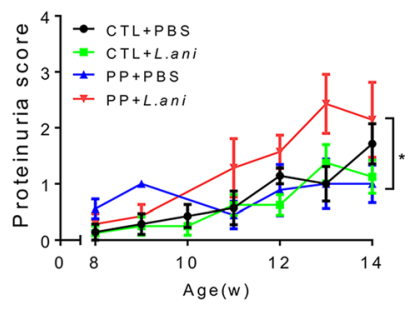

Fig. 3 Identification of a bacterium responsible for the differential effects of vancomycin on control vs. PP MRL/lpr mice. Pregnancy: from 8 to 11 weeks of age. Lactation: from 12 to 14 weeks of age. a Shannon index and observed species of fecal microbiota at the 15 weeks of age $(n=12$ per group). $\mathbf{b}$ Changes of fecal microbiota. Bacterial taxa at the phylum level are shown ( $n=12$ per group). $\mathbf{c}$ Relative abundance of Firmicutes in fecal microbiota of mice with or without vancomycin treatment ( $n \geq 10$ per group). $\mathbf{d}$ Relative abundance of Clostridiales and Erysipelotrichales in fecal microbiota ( $n \geq 10$ per group). e Relative abundance of Lactobacillales and L. animalis in fecal microbiota of mice with or without vancomycin treatment ( $n \geq 10$ per group). $\mathbf{f}$ Level of fecal SCFAs: butyric, proprionic, acetic, and heptanoic acids ( $n \geq 6$ per group). $\mathbf{g}$ Spleen and MLN to body weight ratios at 15 weeks of age ( $n \geq 6$ per group). $\mathbf{h}$ Ratio of anti-DNA IgG to total IgG in mouse serum at 15 weeks of age ( $n \geq 7$ per group). i Level of proteinuria over time ( $n \geq 7$ per group). ${ }^{*} p<0.05,{ }^{* *} p<0.01,{ }^{* * *} p<0.0001$, n.s. not statistically significant

leaky gut [53]. Consistently, we found that in the serum of lupus patients, there were more antibodies against two major microbial antigens-lipopolysaccharide (LPS) and flagellin (Additional file 1: Figure S4A). This suggests increased entry of these antigens from the gut lumen and subsequent triggering of an immune response against them. The concentrations of these two antibodies significantly correlated with each other (Additional file 1: Figure S4B).
As the patient samples were collected after disease onset, it remained unclear whether microbial translocation was a cause or consequence of lupus pathogenesis. We could partially answer this question in lupus-prone mice, as samples could be collected before disease onset. Thus, anti-LPS and anti-flagellin antibodies were examined in MRL/lpr mice at different disease stages as compared to age-matched MRL mice. MRL mice are control mice for MRL/lpr and do not 
develop disease during the time frame that MRL/lpr develop lupus [54]. We found that MRL/lpr mice at late disease stage (lpr-old, 15 weeks of age) had significantly higher IgG levels against the bacterial antigens than age-matched MRL mice (MRL-old) (Additional file 1: Figure S4C). Importantly, however, pre-disease MRL/lpr mice (lpr-young, 7 weeks of age) presented similar levels of these antibodies (Additional file 1: Figure S4C), suggesting that a leaky gut may have occurred prior to disease initiation. Like in human lupus, the levels of the two antibodies highly correlated with each other in lupus-prone mice (Additional file 1: Figure S4D). These results suggest that, rather than a consequence of disease, the leaky gut and subsequent microbial translocation are likely a causative factor in lupus pathogenesis.

In our previous study, vancomycin treatment enhanced barrier function and reduced microbial translocation in naïve MRL/lpr mice [21]. The decreased IgG against LPS confirmed that result (Additional file 1: Figure S4E). Gut microbiota is known as an important factor regulating intestinal barrier function [55]. However, despite the changes of gut microbiota during pregnancy and lactation (Fig. 3a-e), PP mice exhibited similar levels of antibodies against LPS as control mice (Additional file 1: Figure S4E). Vancomycin, possibly through decreasing the bacterial load, decreased the level of anti-LPS antibodies in both control and PP mice (Additional file 1: Figure S4E). This suggests that the exacerbation of lupus disease observed for vancomycin-treated PP mice was not due to microbial translocation. Moreover, while multiple Lactobacillus species have been described as enhancers of intestinal barrier function $[22,56,57], L$. animalis did not change the concentrations of anti-LPS antibodies in either naïve or PP mice (Additional file 1: Figure S4F). This further confirms that the differential response to vancomycin and $L$. animalis in control vs. PP mice cannot be explained by the changes of intestinal barrier function.

\section{Regulation of Treg cells}

Both vancomycin and $L$. animalis worsened lupus disease only in PP mice. However, unlike the effects of vancomycin on IL-17 producing cells (Fig. $2 \mathrm{~b}-\mathrm{d}$ ), L. animalis gavages failed to modulate either DN-T cell (Fig. 4a) or Th17 cell (data not shown) responses in PP mice. We thus focused on the effects of $L$. animalis on splenic Treg cells and circulating anti-inflammatory IL-10 (Fig. 4b), where L. animalis gavages resembled the vancomycin treatment (Fig. 2e, f). More importantly, consistent with the effect of vancomycin (Fig. 2g), L. animalis significantly decreased serum IDO only in PP mice (Fig. 4c). Correspondingly, the bacterial gavage led to significantly lower IDO expression in the spleen (Fig. 4d), but not in the MLN (data not shown). These results suggest that $L$. animalis was able to inhibit IDO, similar to several other Lactobacillus species that have been reported to change IDO expression and activity
[58-60]. Considering the dominant abundance of $L$. animalis upon vancomycin treatment, it is reasonable to believe that the enriched $L$. animalis was responsible for the vancomycin-driven IDO inhibition. As IDO can stimulate Treg cells during the development of autoimmunity [61], these results suggest that vancomycin and $L$. animalis downregulate Treg cells in PP mice through inhibiting IDO.

\section{Involvement of IFNY}

IFN $\gamma$ is known as a pivotal proinflammatory cytokine that promotes lupus disease development [62]. In MRL/ lpr mice, the percentage of IFN $\gamma$ producing $\mathrm{T}$ cells in the spleen increased during the progression of disease (Fig. 5a). Notably, compared to IFN $\gamma^{+/+} \mathrm{MRL} / \mathrm{lpr}$ mice, IFN $\gamma^{+-}$MRL/lpr mice had an extended life span and ameliorated proteinuria, though autoantibody production was not affected [63]. In MRL/lpr mice that had experienced pregnancy and lactation, we found that the effects of vancomycin and $L$. animalis were mostly on lupus nephritis (Figs. 1e and 3i) but not autoantibody production (Figs. 1c and 3h), which coincides with the observation in IFN $\gamma^{+/-}$MRL/lpr mice. In addition, the strong correlation between IDO and IFN $\gamma$ [64] suggests the need to investigate the possible involvement of IFNy. We found that there were more $\mathrm{T}$ cells producing IFNy in PP mice compared to control mice (Figs. 5b and 6c). Further analysis revealed that $\mathrm{CD}^{+} \mathrm{T}$ cells were the major producer of IFNY in both types of mice (Fig. 5b). Interestingly, the proportion of $\mathrm{CD}^{+} \mathrm{IFN}^{+}$cells remained unchanged with or without vancomycin treatment in both control and PP mice (Fig. 5c). In contrast, vancomycin treatment significantly increased the ability of DN-T cells to produce IFN $\gamma$ only in PP mice (Fig. 5c). The serum IFN $\gamma$ level increased accordingly in this group of mice receiving vancomycin (Fig. 5d). Strikingly, oral $L$. animalis gavages recapitulated the effect of vancomycin in PP mice by increasing serum IFN $\gamma$ (Fig. 5e). Similar results were obtained in the MLN (Fig. 5f). These results suggest that vancomycin and $L$. animalis exacerbated lupus nephritis through upregulating IFNY.

Together, our results suggest that the different responses of naive vs. PP MRL/lpr mice to vancomycin or $L$. animalis were irrelevant to microbial translocation; instead, they were a consequence of differential regulation of Treg cells, IDO, and IFNY.

\section{Discussion}

We previously reported that in lupus-prone MRL/lpr mice, oral vancomycin treatment initiated after disease onset ( 9 weeks of age) attenuated lupus manifestations by decreasing splenomegaly and autoantibody production and ameliorating renal damage [21]. The beneficial effect was attributed to the ability of vancomycin to reduce IL17 production from multiple cellular resources including 


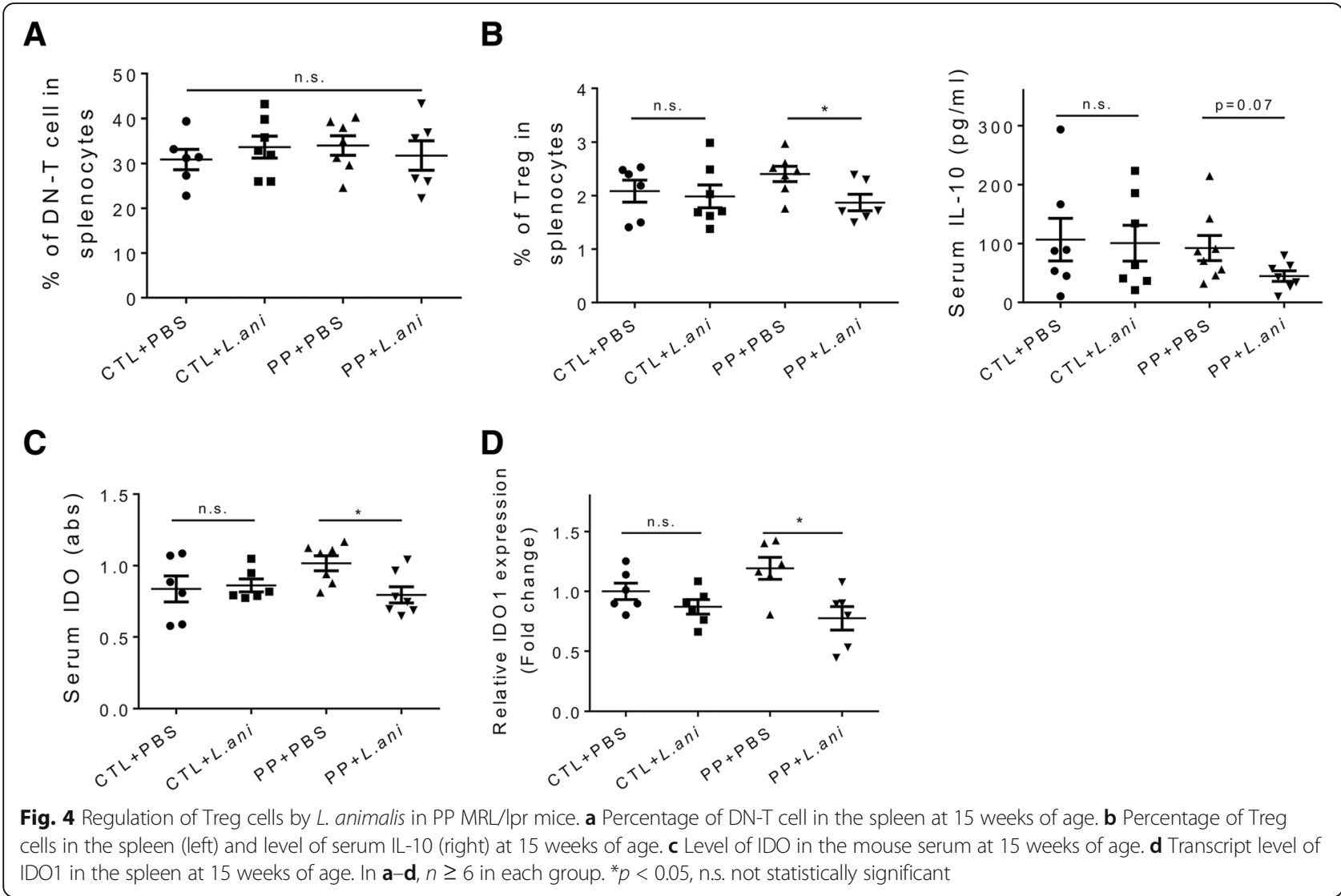

DN-T and Th17 cells and to enhance intestinal barrier function and subsequently inhibiting the translocation of microbial antigens from the gut lumen into the circulation. In this study, our investigation started with a surprising observation that the same antibiotic treatment did not benefit mice which had experienced pregnancy and lactation. Instead, vancomycin significantly worsened splenomegaly and kidney damage in PP MRL/lpr mice, though anti-DNA IgG in the circulation was not changed. Unlike in naïve mice where vancomycin targeted IL-17 producing cells for downregulation [21], the same vancomycin treatment resulted in decreased Treg and B10 cells in PP mice. As a result, the production of anti-inflammatory IL-10 was hampered. At the same time, the production of proinflammatory IFN $\gamma$ from DN-T cells increased in vancomycin-treated PP mice. Therefore, there was a shift of immune balance towards proinflammatory response that could explain how vancomycin exacerbated lupus disease in PP mice.

Vancomycin is not absorbed in the intestine [30]. Hence, the effect of oral vancomycin is limited in the gut lumen without causing systemic side effects. It is known that vancomycin can remove gram-positive bacteria from the gut but spare Lactobacilli [21, 31, 32]. In this study, vancomycin administration again enriched Lactobacillales. One Lactobacillus species, L. animalis, appeared to flourish in the gut microbiota. Isolated from alimentary canal of animals more than 3 decades ago [65], L. animalis has not drawn much attention and there is no evidence suggesting that this bacterium can colonize the human oral, gastrointestinal tract, or vagina [66]. In the Lactobacillaceae family, L. animalis was phylogenetically grouped into the $L$. salivarius clade [67]. A number of species in this clade have been reported to be able to interact with the mammalian host [68], but the information on $L$. animalis is very limited. In the present study, the enrichment of L. animalis in the vancomycin-treated gut prompted us to investigate its role in regulating lupus disease. We found that oral gavages of the bacterium did not affect naïve MRL/lpr mice, but exacerbated lupus nephritis in PP mice. Remarkably, the differential effects of $L$. animalis were highly consistent with the effects of vancomycin on naïve vs. PP mice, suggesting a possible role of $L$. animalis in mediating the action of vancomycin on lupus disease. For example, both vancomycin and $L$. animalis decreased Treg cells and IDO and increased IFNY in PP mice. However, the effects of $L$. animalis and vancomycin were not identical. For instance, vancomycin improved the intestinal barrier function and reduced microbial translocation in both control and PP females; but $L$. animalis failed to exert such improvements. This 
A

C
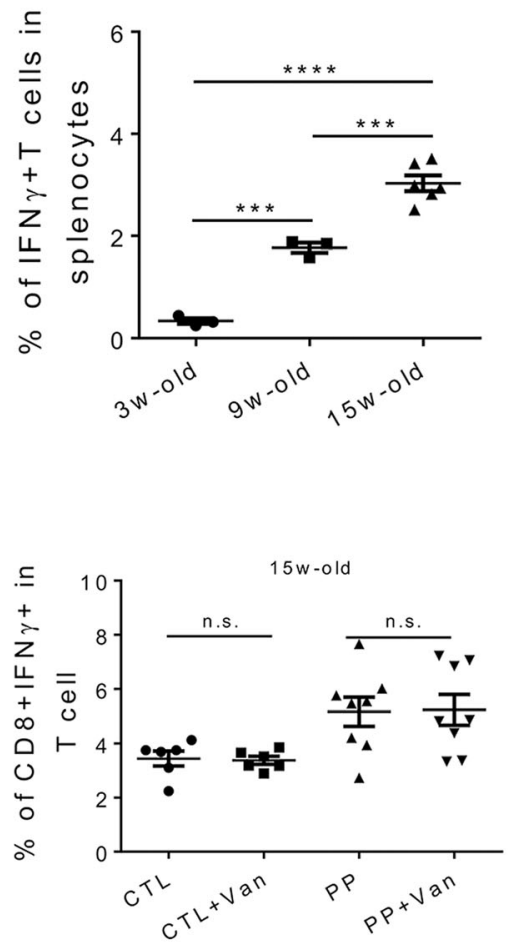

D

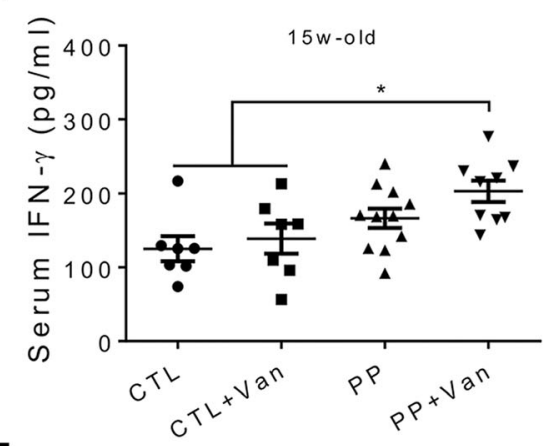

$\mathbf{F}$

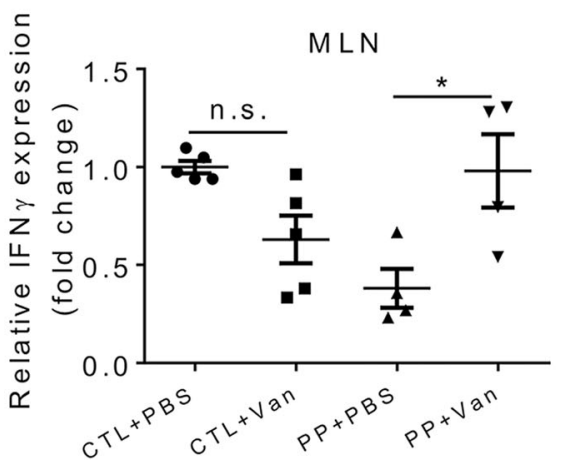

B
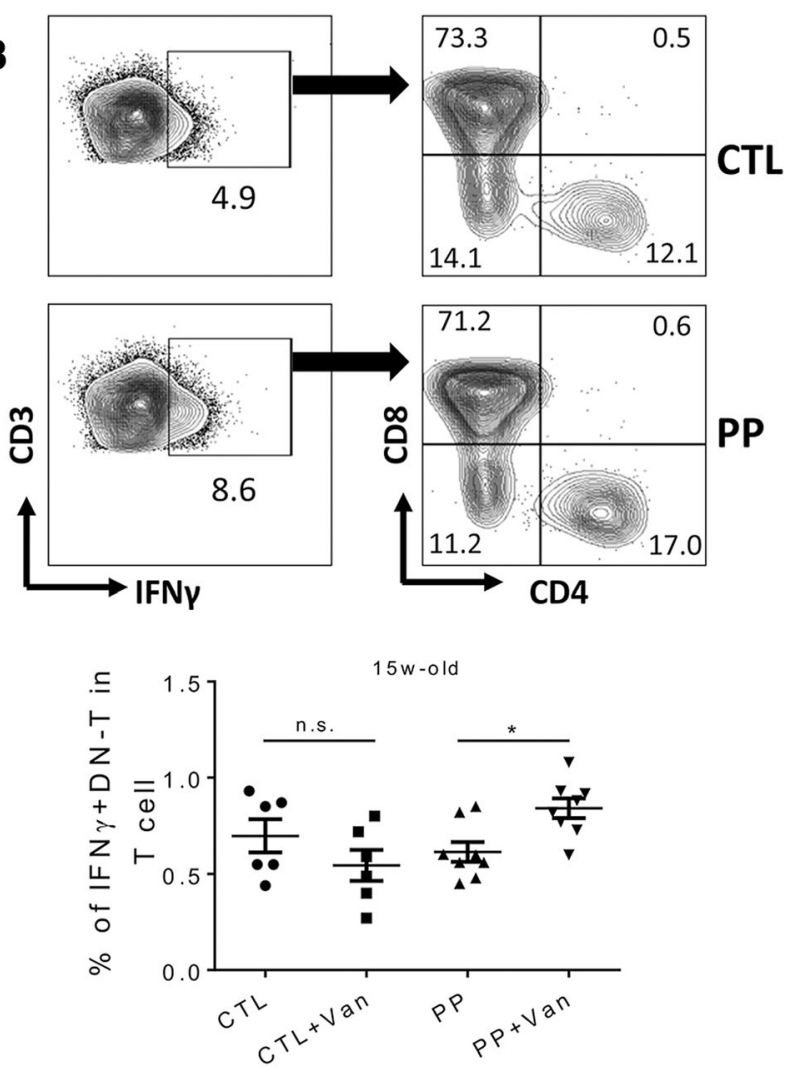

E
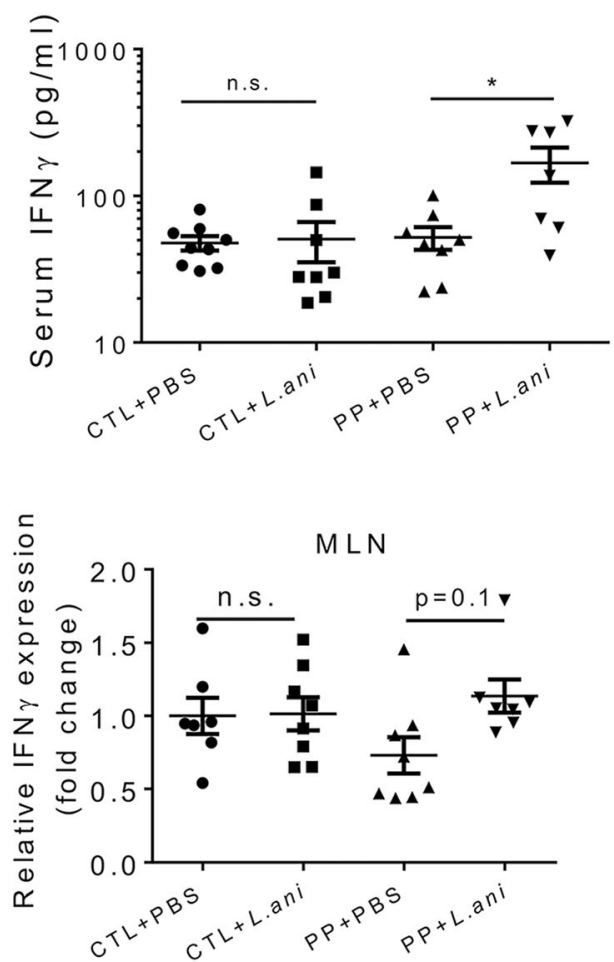

Fig. 5 (See legend on next page.) 
(See figure on previous page.)

Fig. 5 Enhancement of IFNy production by both vancomycin and L. animalis in PP MRL/Ipr mice. a Percentage of IFNy producing T cells in the spleen of mice at different disease stages ( $n \geq 3$ per group). b FACS analysis of IFNy producing T cells. $\mathbf{c}$ Percentage of CD8 ${ }^{+}$IFNY ${ }^{+}$cells and CD4CD8 IFNy ${ }^{+}$cells in splenic T cells at 15 weeks of age ( $n \geq 6$ per group). $\mathbf{d}$ Level of IFNy in the mouse serum at 15 weeks of age ( $n \geq 6$ per group). e Level of IFNy in the mouse serum at 15 weeks of age ( $n \geq 7$ per group). f Transcript level of IFNy in the MLN. ${ }^{*} p<0.05,{ }^{* * *} p<0.001$, ${ }^{* * * *} p<0.0001$, n.s., not statistically significant

is possibly due to the fact that vancomycin can remove many other bacteria in addition to enriching Lactobacilli.

While the diet and housing environment were the same between control and PP MRL/lpr mice, pregnancy, and lactation experiences resulted in significant changes of gut microbiota, affecting microbial diversity, species richness, and microbiota composition. Many gut bacteria are capable of producing SCFAs [28]. However, despite the observed microbiota changes, PP mice had similar levels of the 3 major SCFAs in the feces as naïve mice, suggesting that the differential effects that we have seen were not due to variations of SCFAs. It is widely accepted that lupus patients experiencing pregnancy bear an increased risk of lupus flares $[11,15]$. Sex hormones are believed to trigger the flares, in spite of the tolerant immune response generated during pregnancy [47]. Here, we reported changes of gut microbiota after pregnancy and lactation in lupusprone MRL/lpr mice, but whether these changes would contribute to the triggering of lupus flares requires further investigation. Nonetheless, given the known effects of gut microbiota on host hormones [27, 69], it is reasonable to speculate that gut microbiota variations during and after pregnancy may play a role in triggering lupus disease. Importantly, the gut microbiota-hormone axis may explain why in the current study, the changes of microbiota by vancomycin resulted in different immune responses and subsequently opposite disease outcomes in control vs. PP mice.

In PP mice, we found that oral vancomycin and particularly the enrichment of L. animalis reduced the percentage of Treg cells and lowered IL-10 production. Simultaneously, the production of IFN $\gamma$ increased. Further analysis revealed that the elevated IDO during pregnancy was inhibited by $L$. animalis. This may explain how the regulatory immune response was dampened. IDO is known as a major factor that pregnant individuals rely on to generate immune tolerance for the fetus [48]. It is well established that IDO can induce Treg under normal condition and in various disorders [33, 34]. Notably, Treg cells, IL-10, and IDO are closely linked to IFN $\gamma[64,70]$. Whether the increased IFNY production observed in vancomycin-treated PP mice was due to a dampened regulatory response, or directly originated from the change of IDO, or both, remains unclear.

Interestingly, $L$. animalis is not the only Lactobacillus species that can modulate IDO expression. In simian immunodeficiency virus-infected macaques, the depletion of gut-resident Lactobacillus spp. was associated with increased IDO, and direct supplementation of Lactobacillus-containing probiotics significantly inhibited IDO [60]. In another report, oral feeding of $L$. johnsonii significantly reduced IDO and decreased the incidence of diabetes among BioBreeding diabetes-prone rats [59]. Mechanistically, it was suggested that bacteria-derived $\mathrm{H}_{2} \mathrm{O}_{2}$ from $L$. johnsonii was the cause for the decrease of IDO. On the contrary, feeding of live $L$. reuteri enhanced systemic IDO activity in mice [58].

The generation of autoantibodies against nuclear antigens such as DNA is a hallmark of lupus [8]. However, in the current study, the anti-DNA IgG titer was not changed in PP mice receiving vancomycin or L. animalis treatment. The lowered IL-10 and increased IFN $\gamma$ may be the causes for the exacerbated manifestations in these mice. Coincidently, neither IL- $10^{+/-}$nor $\mathrm{IFN}^{+/-} \mathrm{MRL} /$ lpr had altered autoantibody titers, while the loss of one allele resulted in worsened or attenuated lupus nephritis, respectively [71, 72]. These results suggest that IL-10 and IFN $\gamma$ regulate lupus nephritis in an autoantibodyindependent manner. Additionally, while autoantibodies can promote kidney damage, their importance for the pathogenesis of lupus nephritis is debatable. Indeed, T cells and other immune mediators may be more important in causing the tissue damage [73].

\section{Conclusions}

Our study highlights the changes of gut microbiota structure during pregnancy and the differential effects of the same microbiota-modulating strategies (i.e., vancomycin or $L$. animalis) on disease manifestations in naïve vs. PP MRL/lpr mice. The enzyme IDO, which can be inhibited by specific Lactobacillus spp., appears to be the factor directing the differed immune responses and opposite disease outcomes. The ultimate goal of our research is to identify beneficial as well as pathogenic gut bacterial species and to develop therapeutic strategies that are able to modulate the gut microbiota community towards a beneficial effect. For patients with autoimmune lupus, diet and probiotics are the two relatively easy and acceptable approaches that can potentially improve disease management through modulating the gut microbiota [22, 74]. However, it is challenging to achieve this goal due to the complexity of the disease pathologies, the complexity of gut microbiota, and the differences of gut microbiota communities among individuals. In future investigations, 
we plan to focus on lupus nephritis, the leading cause of mortality in lupus patients, to further delineate the role of gut microbiota in the link between pregnancy and exacerbated lupus.

\section{Methods}

\section{Mice and vancomycin treatment}

MRL/MpJ (MRL, stock number 000486) and MRL/MpJ$\mathrm{Fas}^{l p r} / \mathrm{J}$ (MRL/lpr, stock number 000485) mice were purchased from The Jackson Laboratory (Bar Harbor, ME) and bred and maintained in a specific pathogen-free facility according to the requirements of the Institutional Animal Care and Use Committee (IACUC) at Virginia Tech (Animal Welfare Assurance Number: A3208-01). $\mathrm{CO}_{2}$ was used for euthanasia according to the IACUC protocol. All experiments were performed in accordance with relevant guidelines and regulations. To generate PP mice, 8-week-old female MRL/lpr mice were mated with age-matched male mice. Only the mice delivered at 11 weeks old ( \pm 1 day) were used in the study. Vancomycin $(2 \mathrm{~g} / \mathrm{L})$ was given in the drinking water from 9 weeks old till 15 weeks of age (endpoint). The drinking water containing vancomycin was replenished once a week.

\section{Bacteria culture and gavage}

Lactobacillus animalis (35046) was purchased from ATCC (Manassas, VA) and cultured in Lactobacilli MRS Broth (BD Biosciences) according to the suggested culture method by ATCC. L. animalis was freshly cultured every week and orally gavaged, once a week, to MRL/lpr mice from 9 weeks of age until dissection at 15 weeks.

\section{Microbiota sampling and analysis}

Fecal microbiota samples were obtained by taking the mouse out of the cage and collecting a fecal pellet. Samples were stored at $-80^{\circ} \mathrm{C}$ till being processed at the same time. Sample homogenization, cell lysis, and DNA extraction were performed as previously described [19]. PCR were performed, and purified amplicons were sequenced bidirectionally on an Illumina MiSeq at Argonne National Laboratory. $16 \mathrm{~S}$ rRNA sequencing data were analyzed as described previously [19].

\section{Gas chromatography measurement of SCFAs}

Fecal samples were acidified using phosphoric acid immediately before analysis. Injector settings: temperature $200^{\circ} \mathrm{C}$; carrier, hydrogen; injection mode, split (ratio 2:1). Temperature program: initial temperature of $80^{\circ} \mathrm{C}$ held for $3 \mathrm{~min}$ and then increase temperature at a rate of $6^{\circ} \mathrm{C}$ per minute to $140^{\circ} \mathrm{C}$ and hold for $1 \mathrm{~min}$. The Flame Ionization Detector Settings: temperature, $250^{\circ} \mathrm{C}$; hydrogen flow, $35 \mathrm{ml} /$ minute; air flow, $350 \mathrm{ml} /$ minute; makeup flow (nitrogen), $15 \mathrm{ml} /$ minute; and total makeup (makeup + column flow), $30 \mathrm{ml} /$ minute.

\section{ELISA}

Mouse sera separated after blood clotting were saved at - $20{ }^{\circ} \mathrm{C}$ until use. Human plasma samples were purchased from AllCells (Alameda, CA). For measuring anti-DNA IgG, anti-LPS IgG, and anti-flagellin IgG, high-binding plates were coated overnight at $4{ }^{\circ} \mathrm{C}$ with $100 \mu \mathrm{l}$ of calf thymus DNA $(100 \mu \mathrm{g} / \mathrm{ml}$, Invitrogen $)$, Escherichia coli LPS $(10 \mu \mathrm{g} / \mathrm{ml}$, Sigma), or Salmonella flagellin $(1 \mu \mathrm{g} / \mathrm{ml}$, Enzo Life Sciences), respectively. After sequential incubations on the second day with the samples and secondary antibodies (anti-human IgG-HRP or anti-mouse IgG-HRP, Invitrogen), TMB substrate solution (Thermo Fisher Scientific) was added, followed by the stop solution. Plates were read at $450 \mathrm{~nm}$ absorbance with the SpectraMax M5 microplate reader (Molecular Devices LLC). Mouse serum total IgG was determined with mouse IgG kit (Bethyl Laboratories). Cytokine and enzyme concentrations were determined with mouse IL10, IL-6, IFN $\gamma$ (Biolegend), IL-35 (LifeSpan BioSciences), and IDO (MyBioSource) ELISA kits, respectively, according to the manufacturers' instructions.

\section{Renal function}

Urine was collected weekly starting from disease onset at 9 weeks of age, and all samples were stored at $-20^{\circ} \mathrm{C}$ till analyzed at the same time with a Pierce Coomassie Protein Assay Kit (Thermo Scientific). Proteinuria level was scored as: $0(0-50 \mathrm{mg} / \mathrm{dl}), 1(50-100 \mathrm{mg} / \mathrm{dl})$, $2(100-200 \mathrm{mg} / \mathrm{dl}), 3(200-400 \mathrm{mg} / \mathrm{dl})$, and $4(>400$ $\mathrm{mg} / \mathrm{dl})$. When mice were euthanized at 15 weeks of age, the kidney was fixed in formalin for $24 \mathrm{~h}$, paraffin embedded, sectioned, and stained with periodic acid-Schiff (PAS) at the Histopathology Laboratory at Virginia-Maryland College of Veterinary Medicine. Slides were read with an Olympus BX43 microscope. All the slides were scored in a blinded fashion by a certified veterinary pathologist. Glomerular lesions were graded on a scale of 0 to 3 for each of the following 5 categories: increased cellularity, increased mesangial matrix, necrosis, the percentage of sclerotic glomeruli, and the presence of crescents. Tubulointerstitial lesions were graded on a scale of 0 to 3 for each of the following four categories: presence of peritubular mononuclear infiltrates, tubular damage, interstitial fibrosis, and vasculitis.

\section{Cell isolation and flow cytometry}

Spleens were collected and mashed in $70-\mu \mathrm{m}$ cell strainers with complete media. Red blood cells were lysed with RBC lysis buffer (eBioscience). For surface staining, cells were blocked with anti-mouse CD16/32 (eBioscience), stained with fluorochromeconjugated antibodies, and analyzed with BD FACSAria II flow cytometer (BD Biosciences, San Jose, CA). For intracellular staining, Foxp3 Fixation/Permeabilization kit (eBioscience) was used. Anti-mouse antibodies used in this study include the 
following: CD3-APC, CD4-PE-Cy7, CD8-FITC, IL-10-PerCPCy5.5, IFNY-APC-Cy7, IL-17A-PE, Foxp3-PE, and CD19-APC (Biolegend) and RORyt-BV421 (BD Biosciences). Flow cytometry data were analyzed with FlowJo.

\section{RT-qPCR}

Spleens were homogenized with Bullet Blender homogenizer (Next Advance), and total RNA was extracted with RNeasy Plus Mini Kit (Qiagen) according to the manufacturers' instructions. Genomic DNA was removed by digestion with RNasefree DNase I (Qiagen). Reverse transcription (RT) was performed by using iScript cDNA Synthesis Kit (Bio-Rad). Quantitative PCR (qPCR) was performed with iTaq Universal SYBR Green Supermix (Bio-Rad) and ABI 7500 Fast Real-Time PCR System (Applied Biosystems). Relative quantities were calculated using L32 as the housekeeping gene. Primer sequences for mouse $L 32$ and IDO1 are available upon request.

\section{Statistical analysis}

For the comparison of two groups, unpaired Student's $t$ test was used. For the comparison of more than two groups, one-way ANOVA and Tukey's post-test were used. Results were considered statistically significant when $p<0.05$. All analyses were performed with Prism GraphPad.

\section{Additional file}

Additional file 1: Supplementary Figures S1-S4. (PDF 380 kb)

\section{Abbreviations}

B10: IL-10 producing B cell; DN-T: Double negative T cells; IDO: Indoleamine 2,3-dioxygenase; L. animalis: Lactobacillus animalis; LPS: Lipopolysaccharide; MLN: Mesenteric lymph node; SCFAs: Short chain fatty acids; Th17: T-helper 17 cells; Treg: Regulatory $T$ cell

\section{Acknowledgements}

We thank Husen Zhang for microbiome analysis, Jody Smiley for measuring fecal SCFAs, and Melissa Makris for flow cytometry analysis.

\section{Authors' contributions}

XML and QM designed the experiments. QM performed the experiments. CMR participated in the discussion of results and offered suggestions to analyze IDO in the mechanistic study. XCP, BS, and LA contributed to mouse dissection and sample collection. TEC scored the histopathological slides. $\mathrm{QM}, \mathrm{XML}$, and HW wrote the manuscript. All authors read and approved the final manuscript.

\section{Funding}

This work was supported by the NIH grants AR073240 and AR067418.

\section{Availability of data and materials}

The datasets generated and analyzed during the current study are available in the NCBI SRA accession number SRP182989.

\section{Ethics approval and consent to participate}

This study was carried out in strict accordance with the recommendations in the Guide for the Care and Use of Laboratory Animals of the National Institutes of Health. The protocol was approved by the Institutional Animal
Care and Use Committee (IACUC) of Virginia Tech College of Veterinary Medicine (Animal Welfare Assurance Number A3208-01). For anesthesia and euthanasia, isoflurane and $\mathrm{CO}_{2}$ were used, respectively, according to the IACUC protocol.

Consent for publication

Not applicable.

\section{Competing interests}

The authors declare that they have no competing interests.

\section{Author details}

${ }^{1}$ Department of Biomedical Sciences and Pathobiology, College of Veterinary Medicine, Virginia Tech, Blacksburg, VA, USA. ${ }^{2}$ Translational Biology, Medicine, and Health Graduate Program, Virginia Tech Carilion Research Institute, Virginia Tech, Roanoke, VA, USA. ${ }^{3}$ College of Animal Science, Key Laboratory of Molecular Animal Nutrition, Zhejiang University, Hangzhou, Zhejiang, People's Republic of China. ${ }^{4}$ Edward Via College of Osteopathic Medicine, Blacksburg, VA, USA.

Received: 17 January 2019 Accepted: 5 July 2019

Published online: 16 July 2019

\section{References}

1. Davidson A, Diamond B. Autoimmune diseases. N Engl J Med. 2001;345(5): 340-50.

2. Cooper GS, Bynum MLK, Somers EC. Recent insights in the epidemiology of autoimmune diseases: improved prevalence estimates and understanding of clustering of diseases. Journal of Autoimmunity. 2009;33(3-4):197-207.

3. Khashan AS, et al. Pregnancy and the risk of autoimmune disease. Plos One. 2011;6(5):e19658.

4. Waldorf KMA, Nelson JL. Autoimmune disease during pregnancy and the microchimerism legacy of pregnancy. Immunological investigations. 2008; 37(5-6):631-44.

5. Kumar P, Magon N. Hormones in pregnancy. Niger Med J. 2012;53(4):179-83.

6. Tincani $A$, et al. Pregnancy in patients with autoimmune disease: a reality in 2016. Autoimmunity Reviews. 2016;15(10):975-7.

7. Borchers AT, et al. The implications of autoimmunity and pregnancy. Journal of Autoimmunity. 2010;34(3):J287-99.

8. Tsokos GC. Systemic lupus erythematosus. N Engl J Med. 2011;365(22): 2110-21.

9. Kiriakidou M, et al. Systemic lupus erythematosus. Ann Intern Med. 2013; 159(7):ITC4-1.

10. Almaani S, Meara A, Rovin BH. Update on lupus nephritis. Clin J Am Soc Nephrol. 2017:12(5):825-35.

11. Eudy AM, et al. Effect of pregnancy on disease flares in patients with systemic lupus erythematosus. Annals of the Rheumatic Diseases. 2018. 77(6):855-60.

12. Carp HJA, Selmi C, Shoenfeld $Y$. The autoimmune bases of infertility and pregnancy loss. Journal of Autoimmunity. 2012;38(2-3):J266-74.

13. Chen JS, et al. Pregnancy outcomes in women with rare autoimmune diseases. Arthritis \& Rheumatology. 2015;67(12):3314-23.

14. Moroni G, et al. Maternal outcome in pregnant women with lupus nephritis. A prospective multicenter study. J Autoimmun. 2016;74:194-200.

15. McDonald EG, et al. Monitoring of systemic lupus erythematosus pregnancies: a systematic literature review. Journal of Rheumatology. 2018; 45(10):1477-90.

16. Kahrstrom, C.T. N. Pariente, and U. Weiss, Intestinal microbiota in health and disease. Nature, 2016. 535(7610): p. 47-47.

17. Mu QH, Zhang HS, Luo XM. SLE: Another autoimmune disorder influenced by microbes and diet? Frontiers in Immunology. 2015;6:808.

18. Hevia, A., et al., Intestinal dysbiosis associated with systemic lupus erythematosus. Mbio, 2014. 5(5).

19. Zhang HS, et al. Dynamics of gut microbiota in autoimmune lupus. Applied and Environmental Microbiology. 2014:80(24):7551-60.

20. Luo XM, et al. Gut microbiota in human systemic lupus erythematosus and a mouse model of lupus. Applied and Environmental Microbiology. 2018; 84(4):e02288.

21. Mu Q, et al. Antibiotics ameliorate lupus-like symptoms in mice. Sci Rep. 2017;7(1):13675 
22. Mu Q, et al. Control of lupus nephritis by changes of gut microbiota. Microbiome. 2017;5(1):73.

23. Vieira SM, et al. Translocation of a gut pathobiont drives autoimmunity in mice and humans. Science. 2018;359(6380):1156-60.

24. $\mathrm{Mu} \mathrm{Q}$, et al. Leaky gut as a danger signal for autoimmune diseases. Front Immunol. 2017:8:598.

25. Zegarra-Ruiz DF, et al. A diet-sensitive commensal Lactobacillus strain mediates TLR7-dependent systemic autoimmunity. Cell Host Microbe. 2018.

26. Greiling TM, et al. Commensal orthologs of the human autoantigen Ro60 as triggers of autoimmunity in lupus. Science Translational Medicine. 2018; 10(434):eaan2306.

27. Li JY, et al. Sex steroid deficiency-associated bone loss is microbiota dependent and prevented by probiotics. Journal of Clinical Investigation. 2016;126(6):2049-63.

28. Morrison DJ, Preston T. Formation of short chain fatty acids by the gut microbiota and their impact on human metabolism. Gut Microbes. 2016; 7(3):189-200

29. Nuriel-Ohayon M, Neuman H, Koren O. Microbial changes during pregnancy, birth, and infancy. Frontiers in Microbiology. 2016;7.

30. Rao $\mathrm{S}$, et al. Systemic absorption of oral vancomycin in patients with Clostridium difficile infection. Scand J Infect Dis. 2011:43(5):386-8

31. Robinson CJ, Young VB. Antibiotic administration alters the community structure of the gastrointestinal micobiota. Gut Microbes. 2010;1(4):279-84.

32. Murphy EF, et al. Divergent metabolic outcomes arising from targeted manipulation of the gut microbiota in diet-induced obesity. Gut. 2013;62(2):220-6.

33. Baban $\mathrm{B}$, et al. IDO activates regulatory $T$ cells and blocks their conversion into Th17-Like T cells. Journal of Immunology. 2009;183(4):2475-83.

34. Sharma MD, et al. Indoleamine 2,3-dioxygenase controls conversion of Foxp3(+) Tregs to TH17-like cells in tumor-draining lymph nodes. Blood. 2009;113(24):6102-11.

35. Munn DH. Indoleamine 2,3-dioxygenase, Tregs and cancer. Current Medicinal Chemistry. 2011;18(15):2240-6.

36. Isenberg DA, et al. Fifty years of anti-ds DNA antibodies: are we approaching journey's end? Rheumatology (Oxford). 2007;46(7):1052-6.

37. Kimura A, Kishimoto T. IL-6: regulator of Treg/Th17 balance. Eur J Immunol. 2010;40(7):1830-5

38. Tackey E, Lipsky PE, Illei GG. Rationale for interleukin-6 blockade in systemic lupus erythematosus. Lupus. 2004;13(5):339-43.

39. Crispin JC, et al. Expanded double negative T cells in patients with systemic lupus erythematosus produce IL-17 and infiltrate the kidneys. I Immunol. 2008;181(12):8761-6.

40. Martina MN, et al. Double negative (DN) alphabeta T cells: misperception and overdue recognition. Immunol Cell Biol. 2015;93(3):305-10.

41. Korn T, et al. IL-17 and Th17 cells. Annu Rev Immunol. 2009;27:485-517.

42. Arellano B, Graber DJ, Sentman CL. Regulatory T cell-based therapies for autoimmunity. Discov Med. 2016;22(119):73-80.

43. Eisenstein EM, Williams CB. The T(reg)/Th17 cell balance: a new paradigm for autoimmunity. Pediatr Res. 2009;65(5 Pt 2):26R-31R.

44. Mauri C, Menon M. Human regulatory B cells in health and disease: therapeutic potential. J Clin Invest. 2017;127(3):772-9.

45. Choi J, et al. IL-35 and autoimmunity: a comprehensive perspective. Clin Rev Allergy Immunol. 2015;49(3):327-32.

46. Lee CC, et al. Macrophage-secreted interleukin-35 regulates cancer cell plasticity to facilitate metastatic colonization. Nat Commun. 2018;9(1):3763.

47. Guleria I, Sayegh MH. Maternal acceptance of the fetus: true human tolerance. J Immunol. 2007;178(6):3345-51.

48. Kudo $Y$. The role of placental indoleamine 2,3-dioxygenase in human pregnancy. Obstet Gynecol Sci. 2013;56(4):209-16.

49. Koren $\mathrm{O}$, et al. Host remodeling of the gut microbiome and metabolic changes during pregnancy. Cell. 2012;150(3):470-80.

50. Azzouz D, et al. Lupus nephritis is linked to disease-activity associated expansions and immunity to a gut commensal. Ann Rheum Dis. 2019.

51. Fasano A. Leaky gut and autoimmune diseases. Clin Rev Allergy Immunol. 2012;42(1):71-8

52. Manfredo Vieira S, et al. Translocation of a gut pathobiont drives autoimmunity in mice and humans. Science. 2018;359(6380):1156-61.

53. Shi $L$, et al. The SLE transcriptome exhibits evidence of chronic endotoxin exposure and has widespread dysregulation of non-coding and coding RNAs. PLoS One. 2014;9(5):e93846.

54. Richard ML, Gilkeson G. Mouse models of lupus: what they tell us and what they don't. Lupus Science \& Medicine. 2018;5(1):e000199.
55. Kelly JR, et al. Breaking down the barriers: the gut microbiome, intestina permeability and stress-related psychiatric disorders. Front Cell Neurosci. 2015;9:392.

56. Eun CS, et al. Lactobacillus casei prevents impaired barrier function in intestinal epithelial cells. APMIS. 2011;119(1):49-56.

57. Sultana R, McBain AJ, O'Neill CA. Strain-dependent augmentation of tightjunction barrier function in human primary epidermal keratinocytes by Lactobacillus and Bifidobacterium lysates. Appl Environ Microbiol. 2013; 79(16):4887-94.

58. Forsythe P, Inman MD, Bienenstock J. Oral treatment with live Lactobacillus reuteri inhibits the allergic airway response in mice. Am J Respir Crit Care Med. 2007;175(6):561-9.

59. Valladares $\mathrm{R}$, et al. Lactobacillus johnsonii inhibits indoleamine 2,3dioxygenase and alters tryptophan metabolite levels in BioBreeding rats. FASEB J. 2013;27(4):1711-20.

60. Vujkovic-Cvijin I, et al. Gut-resident Lactobacillus abundance associates with IDO1 inhibition and Th17 dynamics in SIV-infected macaques. Cell Rep. 2015;13(8):1589-97.

61. Lippens C, et al. IDO-orchestrated crosstalk between pDCs and Tregs inhibits autoimmunity. Journal of Autoimmunity. 2016;75:39-49.

62. Pollard KM, et al. Interferon-gamma and systemic autoimmunity. Discov Med. 2013;16(87):123-31

63. Balomenos D, Rumold R, Theofilopoulos AN. Interferon-gamma is required for lupus-like disease and lymphoaccumulation in MRL-Ipr mice. J Clin Invest. 1998;101(2):364-71.

64. Heyes MP. Relationship between interferon-gamma, indoleamine-2,3dioxygenase and tryptophan. FASEB J. 1991;5(14):3003-4.

65. Dent VE, Williams RAD. Lactobacillus-animalis sp-nov, a new species of Lactobacillus from the alimentary canal of animals. Zentralblatt Fur Bakteriologie Mikrobiologie Und Hygiene I Abteilung Originale CAllgemeine Angewandte Und Okologische Mikrobiologie. 1982;3(3):377-86.

66. O'Callaghan J, O'Toole PW. Lactobacillus: host-microbe relationships. Between Pathogenicity and Commensalism. 2013;358:119-54.

67. Zhang ZG, et al. Phylogenomic reconstruction of lactic acid bacteria: an update. Bmc Evolutionary Biology. 2011;11:1.

68. Neville BA, O'Toole PW. Probiotic properties of Lactobacillus salivarius and closely related Lactobacillus species. Future Microbiology. 2010;5(5):759-74.

69. Kwa M, et al. The intestinal microbiome and estrogen receptor-positive female breast cancer. Inci-Journal of the National Cancer Institute. 2016; 108(8).

70. Panduro M, Benoist C, Mathis D. T-reg cells limit IFN-gamma production to control macrophage accrual and phenotype during skeletal muscle regeneration. Proceedings of the National Academy of Sciences of the United States of America. 2018;115(11):E2585-93.

71. Yin ZN, et al. IL-10 regulates murine lupus. Journal of Immunology. 2002; 169(4):2148-55.

72. Balomenos D, Rumold R, Theofilopoulos AN. Interferon-gamma is required for lupus-like disease and lymphoaccumulation in MRL-Ipr mice. Journal of Clinical Investigation. 1998;101(2):364-71.

73. Suarez-Fueyo A, Bradley SJ, Tsokos GC. T cells in systemic lupus erythematosus. Current Opinion in Immunology. 2016;43:32-8.

74. Vieira SM, Pagovich OE, Kriegel MA. Diet, microbiota and autoimmune diseases. Lupus. 2014;23(6):518-26.

\section{Publisher's Note}

Springer Nature remains neutral with regard to jurisdictional claims in published maps and institutional affiliations.

\section{Ready to submit your research? Choose BMC and benefit from}

- fast, convenient online submission

- thorough peer review by experienced researchers in your field

- rapid publication on acceptance

- support for research data, including large and complex data types

- gold Open Access which fosters wider collaboration and increased citations

- maximum visibility for your research: over $100 \mathrm{M}$ website views per year

At $\mathrm{BMC}$, research is always in progress.

Learn more biomedcentral.com/submissions 